\title{
Immunotherapeutic Potential of Plasmodium Against Cancer by Inducing Immunomodulation
}

\author{
Aqsa Riaz ${ }^{1,3}$ Awais Ali ${ }^{1,2} \quad$ Usman Babar ${ }^{2,3,4} \quad$ Usama Arshad $^{2,3,4}$ Muhammad Asad Ullah ${ }^{1,3,4}$ \\ Ali Usman ${ }^{1,2}$ Mirza Sabuktgeen Baig ${ }^{1,2}$ Muhammad Faizan Manzoor ${ }^{1,2}$ Usama Zahir ${ }^{1,2}$. \\ 1.Institute of Molecular Biology and Biotechnology (IMBB), University of Lahore, Pakistan \\ 2.Centre for Research in Molecular Medicine (CRiMM), University of Lahore, Pakistan \\ 3.University Institute of Diet and Nutritional Sciences (UIDNS), University of Lahore, Pakistan \\ 4.Centre of Agricultural Biochemistry and Biotechnology (CABB), University of Agriculture, Faisalabad \\ 5.Centre of Advanced Studies, Agriculture and Food Security (CAS-AFS), University of Agriculture,
} Faisalabad, Pakistan

\begin{abstract}
Cancer is one of the most death leading disease across the globe because of the unavailability of innocuous treatment strategy for cancer control so far but while having comparative study with malaria, it was found that there is some resemblance within the physiological conditions of the body as well as some antagonistic effects. Plasmodium is a parasitic microbe that is familiar for causing malaria within the animals and by thorough study, the immunotherapeutic potential of this organism was found against cancer as it consists of some tumorassociated antigens (TAAs) that favors the body in combating tumor by inducing immunomodulation and causing certain advantageous physiological changes.
\end{abstract}

Keywords: Chimeric Antigen Receptor, Genetically Attenuated Sporozoites, Glycosyl Phosphatidyl Inositol, Hepatocellular Carcinoma, Interdomain, Interferon, Lewis Lung Cancer, Pathogen-associated Molecular Patterns, Tumor-associated Antigens, Terminal deoxynucleotidyl Transferase-dUTP Nick End Labeling

DOI: $10.7176 /$ ALST/70-03

\section{Introduction}

Plasmodium is a protozoan that belongs to the genus of parasitic alveolates which feed on their hosts are known for their infectious cause i.e. Malaria. Plasmodium is representative of family; Plasmodiidae, Order; Haemosporida, Class; Aconoidasida, Phylum; Apicomplexa and Domain' Eukaryota by its taxonomy and characteristics. There are almost 200 species of plasmodium that have been identified so far. The parasite was $1^{\text {st }}$ diagnosed and identified over a century ago, in 1880 by Charles Louis Alphonse Laveran within malaria patients. ${ }^{[1]}$ Cancer is one of the most fatal health disasters throughout the world. Depending upon the type and nature of tumor, it can be benign (Acute or non-cancerous) or malignant (Chronic or cancerous). Term 'Cancer' is basically coined for malignant tumor that is highly lethal as it metastasize, retains inside body for prolonged period and ultimately leads to the death. ${ }^{[2]}$ Lung cancer is the most common among all the cancer types throughout the world. According to a survey, it was reported that increment of around 1.8 Million cases was recorded per year, similarly, deaths of 1.6 Million cases shows the increased mortality rate due to this disease. ${ }^{[3]}$ Different approaches such as chemotherapy, radiotherapy and surgery etc have been invented and improved for cancer cure and control but still the prognosis remains dissatisfying. Therefore, the development of immunotherapy or therapeutics is mandatory in order to control or getting rid of cancer. ${ }^{[4]}$

The development of therapeutic vaccination was the major aspect of immunotherapy that was passively induction or elicitation of the host immune system in response to the tumor-associated antigens (TAAs). ${ }^{[5]}$ But the recent studies and researches have claimed the presence of some parasites like Plasmodium may play a role in getting rid of immunosupression and with the enhanced immune system, it helps in the cancer growth inhibition. ${ }^{[6]}$ Hence, the parasites can also be one of the therapeutic approach for cancer treatment in near future. It has been reported that the method of immunotherapy was developed in order to cure the lung cancer by eliciting the immune response of the patient's body with the help of some immunostimulants that were specific as well as non-specific to tumor antigens like mucin-1 (MUC1) and melanoma-associated antigen-3. As the cancer weakens host immunity by causing immunosuppression in body. ${ }_{[7]}$

By last century, Malaria was supposed to be very problematic as it causes hyperthermia, high fever, and also led to death in several cases due to failure of homeostasis. The etiology of disease was found as presence of an intracellular parasite, Plasmodium, that was responsible for causing human malaria parasitic infection. ${ }^{\left[{ }^{[1}\right.}$ In $21^{\text {st }}$ century, the clinical significance of this malarial infection has been studied thoroughly and it has been reported that the infection is responsible for the enhancement of various immunological processes such as proliferation of T-cells, maturation induction in dendritic cells (DC) ${ }^{[9]}$, increased production of Interferon (IFNc) and activation of cell mediated immunity that involves Natural killer (NK) cells, NKT-cells and cytotoxic Tcells. ${ }^{[10]}$ Such fluctuation in the body immune response produces the favorable conditions that are proved to be advantageous in confronting different types of the clinical cancers. ${ }^{[1]}$ Clinically, a malarial symptom, 
hyperthermia, has also been observed as a significant body condition for combating and treating few cancer types. $^{[12]}$ Therefore, the malarial parasite, Plasmodium, due to its clinical importance in cancer treatment has been considered as a therapeutic agent. The parasite was analyzed within mouse model and for the confirmation, murine lewis lung cancer (LLC) was treated with it. The immunological mechanisms as well as the antitumor effects of this therapeutic agent were studied. It was reported that the metastasis and cancer growth was controlled by the plasmodium. The antitumor effect was caused due to the induction of dynamic innate and adaptive immunity that developed in reponse to the uncontrolled or abnormal neoplastic growth. ${ }^{[13]}$

$85-90 \%$ cases of Hepatocellular Carcinoma (HCC) were reported according to survey out of all the types of liver cancers. It is the $3^{\text {rd }}$ most fatal cancer type throughout the world. ${ }^{[14]}$ In 2012 , around 782,500 new cases and approximately 745,500 deaths due to liver cancer were recorded. ${ }^{[15]}$ At the initial cancer stages, AFP, $\alpha$-fetal protein, from dendritic cells or glypican III (GPC3) derived vaccines were tested and the cellular mediated immune response by cytotoxic T-cells was activated in HCC patients. As a result of that, a huge decline in the mortality rate was observed. ${ }^{[16]}$ By performing further clinical trials, more encouraging results were examined for combating the HCC. ${ }^{[17]}$

It has been acknowledged that the tumor growth can be suppressed by some pathogenic infections as they comprised of the counter ability. ${ }^{[18]}$ Genomic DNA, haemozoin ${ }^{[19]}$, pathogen-associated molecular patterns (PAMPs) and glycosylphosphatidylinositol anchors (GPI) ${ }^{[20]}$ are the major constituents of Plasmodium parasite that are usually detected by the host's innate immune system. Thus, dendritic cells, macrophages, NK-cells, NKT-cells, $\gamma \delta$-T-cells, cytotoxic T-cells and helper T-cells are activated at the initial stage of cancer i.e. bloodstage (erythrocytic stage). ${ }^{[21]}$ Hence, it was reported that the enhancement in the host immune system due to a pathogenic infection also confront the antitumor immunity. GPC3 is one of the most significant tumor-associated antigens (TAAs) that is related to hepatocellular carcinoma (HCC) and its cytotoxic T-lymphocytes (CTL) are found to be present in both mice and the patients. ${ }^{[22]}$ This TAA, GPC3 Ag was used in the development of certain peptide vaccines that showed high survival rate in patients. ${ }^{[16]}$ The application of GPC3-peptide vaccine to the patients that have already received radiofrequency ablation (RFA) surgery or therapy showed the improvement in their survival rate upto a year. ${ }^{[23]}$ Moreover, chimeric antigen receptor (CAR) T-cells have been developed that are targeted by GPC3 antigen in order to get the therapeutics for treatment. ${ }^{[24]}$ It has been reported that GPC3 is an ideal TAA for the immunotherapy of HCC due to its distinct immunological characteristics. ${ }^{[25]}$

\section{Infection Mechanism of Plasmodium}

Malaria infection is caused in the host by the injection of Plasmodium within the blood stream of host at sporozoites stage by the help of female Anopheles mosquito's bite. The mosquito acts as a vector for this infectious agent. Characteristics of Plasmodium species while causing malaria are shown in Table 1. It is persumed that a single sporozoite is enough for causing infection in host but the number injected by the mosquito while having a meal varies from a few dozens to thousands. By clinical diagnosis of the malaria patients, it has been reported that sporozoite load, short incubation period and serious symptoms for malaria are caused due to a mosquito bite. The sporozoites stays in blood stream for an hour and then invades liver of host. ${ }^{[26]}$ The sporozoites either invades Kuppfer cells of liver, failed to develop themselves in it and die soon or they enter hepatocytes and starts to reproduce asexually through 'Exo-erythrocytic Schizogonic cycle'.

In liver, trophozoite seems like mono-nucleated round body within host cytosol for the nutrient uptake. With the passage of time, it develops and replicates by asexual reproduction and turned into a mature, mutinucleated parasite i.e. Schizont. It is approximately 30-70 micrometres in size and doesn't exhibit any pigment due to absence of hemoglobin in hepatocytes. It starts to occupy entire cellular cytoplasm for over a week to two and then ultimately release a wide range $(2,000-30,000)$ of merozoites. It is the final stage of the life cycle of plasmodium in which the released merozoites starts to invade RBCs. The mechanism is similar in all the experimented plasmodium species such as Plasmodium falciparum, Plasmodium ovale, Plasmodium vivax and Plasmodium malariae. ${ }^{[2]}$ Two of these species $P$. Vivax and $P$. Ovale also shows an additional stage in their life cycle in which they stays into liver for a variable time period in latent stage and are known as hypnozoites. ${ }^{[28]}$ 
Table 1: Characteristics of Malaria Infection in Human Plasmodium Species

\begin{tabular}{l|cccc}
\hline Infection Characteristics & $\boldsymbol{P}$. vivax & $\boldsymbol{P}$. ovale & $\boldsymbol{P}$. malariae & $\boldsymbol{P}$. falciparum \\
\hline Pre-erythrocytic Cycle & 8 days & 9 days & 13 days & $5-6$ days \\
Erythrocytic Cycle & $48 \mathrm{~h}$ & $50 \mathrm{~h}$ & $72 \mathrm{~h}$ & $48 \mathrm{~h}$ \\
Pre-patent period & $10-13$ days & $10-14$ days & $15-16$ days & $9-10$ days \\
Incubation Period & $12-17$ days & $16-18$ days & $18-40$ days & $9-14$ days \\
No. of Schizont & $\sim 10,000$ & $\sim 15,000$ & $\sim 2,000$ & $\sim 40,000$ \\
Hypozoites & + & + & - & - \\
Average Parasitemia & 20,000 & 9,000 & 6,000 & $50,000-500,000$ \\
Maximum Parasitemia & 50,000 & 30,000 & 20,000 & $2,500,000$ \\
Severity of Initial paroxysms & Moderate & Mild & Moderate & Severe \\
Typical Symptom Duration & $3-8$ weeks & $2-3$ weeks & $3-24$ weeks & $2-3$ weeks \\
Maximum Infection Duration & $5-8$ years & $12-20$ months & $20-50$ years & $6-17$ months \\
Anemia & Moderate & Mild & Moderate & Severe \\
Other Complications & - & - & Renal & Cerebral \\
\hline
\end{tabular}

Identification of Tumor-associated Antigens from Plasmodium

In malaria parasite, Plasmodium falciparum, glycosylphosphatidylinositol (GPI) was examined and reported as a tumor-associated antigen (TAA) because of its induction role in proinflammatory responses within host via Tolllike its receptor (TLR-2) in MyD88 (myeloid Differentiation) mediated signaling pathway. ${ }^{[29]}$ Hemin detoxification produces a byproduct within plasmodium i.e. Hemozoin, a biocrystalline substance that regulates the activation of immune system of host via TLR-9. ${ }^{[30]}$ Along with certain model antigens, it also showed the potent adjuvant effect. A tumor-specific effect or response can be facilitated by inbuilt adjuvant. By taking up with a vaccine, this plasmodium component can be an ideal adjuvant as it is performing a function against tumor hence, it can also be a TAA for the tumor-specific vaccine development. ${ }^{[31,32]}$ The TAAs reported so far are mentioned in Table 2.

Table 2: Tumor-associate Antigens (TAA) and their Respective Functions

\begin{tabular}{l|l}
\hline TAAs from Plasmodium & Functions \\
\hline GPI & $\begin{array}{l}\text { Induction of Pro-inflammatory Responses } \\
\text { Hemozoin }\end{array}$ \\
\hline Other TAAs & \multicolumn{2}{|l}{ Activation of Innate Immune System } \\
\hline AFP (a-Fetal Protein) & Oncofetal Antigen \\
CEA (Carcinoembryonic Ag) & Oncofetal Antigen \\
MUC1 (Mucin-1) & Overexpression in Tumor Cells \\
GPC3 (Glypican-3) & Role in Cell Division and Growth Regulation \\
\hline
\end{tabular}

\section{Antitumor Activity of Plasmodium against Lewis Lung Cancer}

It was believed that malaria caused by Plasmodium yoelii 17XNL shows some antitumor activity within erythrocytic stage hence, an experiment was performed on two groups of mice and the similar number of infected as well as plain LLC cells of mice were injected in model organisms for the evaluation of the effect of infection over LLC growth and metastasis. It was observed that within the malarial infectious period, the suppression in the tumor growth, volume and metastasis was developed while in the mice having just LLC healthy cells showed tumor growth. After a month of the inoculation of erythrocytes (both LLC and infected LLC), the changes with the passage of time in growth and metastasis were evaluated and it was reported that the LLC cells divided and metastasized at constant rate while the rate of growth and metastasis in plasmodium infected LLC cells reduced to a negligible amount i.e. 1/10. The trial was conducted on the aggresively metastasizing models that were produced by injecting LLC tumor cells. ${ }^{[33]}$

The crucial cause of death among the cancer patients is distant metastases. ${ }^{[34]}$ Hence, two different treatment strategies (Surgery and malarial infection) were applied together for the verification of anti-metastatic activities in host. At the initial stage, primary tumor was removed surgically and after 5 days, plasmodium was infected in half of the mice models and rest were left uninfected. The plasmodium infected mice tolerated the primary tumor after surgery and survived longer than the other ones. These outcomes represented that the malarial parasite, plasmodium, exhibit the capability to counter the late steps of metastasis that usually develops in host with the invasion of metastatic tumor cells from the blood stream. ${ }^{[13]}$

Improvement in LLC Host Physiological Processes by Malaria and their Verification by Tumor Markers Malaria is a pathogenic infection that is usually caused by Plasmodium parasite that plays a significant role in enhancing the host's physiological processes against the tumor like cell proliferation, apoptosis and angiogenesis. 
Tumor staining with Ki67, a proliferation marker, was examined within the malarial infected LLC mice model and the suppression in the tumor cell proliferation was reported in it. That suppression act as a signaling for the apoptosis activation within the tumor cells due to plasmodium presence while no such change was observed in LLC mice models. After 17 days, the terminal deoxynucleotidyl transferase-dUTP nick end labeling (TUNEL) assay of the tumors was performed. In order to check the effect of proliferation decrease and apoptosis increase whether related to vascular pruning in the tumor blood vessels. ${ }^{[35]}$ Similarly, CD31 was also stained over the endothelial cells of blood vessels in order to examine the vasculature of the tumor within mice with LLC and both (LLC and plasmodium) and the suppression of angiogenesis was observed in the infected group.

Table 3: Improvements in Host System by Plasmodium Infection

\begin{tabular}{|c|c|}
\hline Improved Physiological Processes by Malaria & Improved Immunological Processes by Malaria \\
\hline $\begin{array}{ll}\text { - } & \text { Tumor Cell Proliferation } \\
\text { - } & \text { Apoptosis } \\
\text { - } & \text { Angiogenesis }\end{array}$ & $\begin{array}{ll}\text { - } & \text { DCs Maturation } \\
\text { - } & \text { Cytotoxicity Induction in NK-Cells } \\
\text { - } & \text { Increased TH1-type Cytokines Production }\end{array}$ \\
\hline
\end{tabular}

\section{Mechanism of Plasmodium Antitumor Activity}

The pre-activated antibodies (Ab), non-tumor specific as well as TAA-specific immune responses (Both Cellular and Humoral) were suppressed due to tumor growth but recovered with the infection of malaria parasite and plays a significant role in inhibition of tumerigenesis at its earlier or initial stage. With the invasion of malaria infection within the tumor exhibiting cells/host, a series of innate as well as adaptive immune responses are enhanced and induced that act against the tumor. Certain components of plasmodium are pronounced to be of purpose while developing a TAA-specific vaccine. ${ }^{[30]}$

\section{Induction of Adaptive Immunity by Tumor Associated Antigens}

Due to weak immunogenic ability of LLC cells, no potential immune response could be generated by the body. The major reason of that is resemblance of neoplastic LLC cells with the normal body cells. Therefore, it is very challenging to study and identify the antigen (Ag) specificity that have induced the immune response (IR) within the host body containing LLC cells. ${ }^{[36]}$ A recombinant LLC cell line (LLC-MUC1) expressing mucin-1 glycoprotein was developed for the evaluation of TAA-specific immune responses that were induced by invasion of malaria infection.

For further confirmation of the origin of specificity of induced immune response whether it is TAA or any other self-antigen of mouse. A cross-species cell line (LLC-MUC1) was developed by inserting an exogenous mucin-1 gene from human into cancerous cell line of mouse. It was reported that the cell line having human mucin-1 gene showed greater immunogenecity within mice as compared to the recombinant cell line. Due to the clear observation of epitope of human MUC1, it would now be easy to interpret the distinctions in the immune responses among different cell lines under observation that were TAA-specific.

\section{Maturation Induction in Dendritic Cells}

There was not much variation observed among the parent LLC-cells and recombinant LLC-MUC1 cells in their phenotypic traits as well as growth under both (in vivo and in vitro) conditions. Here, the major role of dendritic cells (DCs) was found that was associated with the initiation and maintenance of antigen-specific immunity. ${ }^{[37]}$ In the malarial infected (Tumor-bearing) mice, the proportion and maturation of DCs along with CD11C (Integrin) was analyzed within the lymph node and it was reported that the percentage of DCs + CD11C was increased due to the antitumor activity and enhancement in immune response at the earlier stage.

The expression of clusters of differentiation (CD80 and CD86) located on the dendritic cells was also upregulated due to increase in CD11C and DCs. That change was reported not to be found in uninfected tumor LLC cells that might be due to the suppression of immune system. The functions of DCs (tumor-specific T-cells recruitment and cross-priming) were checked whether they are affected with the enhancement in dendritic cells due to malarial infection. ${ }^{[38]}$ Hence, the LLC-MUC1 cell line was analyzed by enzyme-linked immunospot (ELISPOT) for the production of TAA-specific granzyme B and IFN-c and significant increment was reported.

\section{Activation of Cell-Mediated Immunity}

The significant increase in the number of helper T-cells (CD4+) and Cytotoxic T-cells (CD8+) and their accumulation was also noticed within the tumor cells due to malarial infection that was confirmed by comparison. Hence, the cytotoxicity was also increased due to activity of increased cytotoxic T-cells. Likewise, the increased production of IFN-c that was specific to mucin-1 from the plasmodium infected LLC-MUC1 cell line took place in the spleen along with granzyme B-secreting cells and also showed systemic antitumor immunity. Proliferation of tumor-specific T-cells and TAA-specific proliferative ability was found to be increased due to malaria parasite infection in tumor cells that was responsible for the induction of systemic 
antitumor immune response.

\section{Induction of Cytotoxicity in Natutal Killer Cells}

The cytotoxic activity of natural killer cells is included in adaptive immunity after the invasion of an antigen withn body following innate immunity confronted by the cytokines and Th1-type lymphocytes production i.e. TNF- $\alpha$ and IFN-c. They usually respond rapidly but in case of tumor (LLC), the host immune system had been compromised so no such action was happening. ${ }^{[39]}$ But with the malarial infection, the rapid increase in the TNF$\alpha$ and IFN-c production levels was observed right after three days from the infection. Due to which potential enchancement in lytic activity of NK-cells was reported. Though the NK-cells percentage in the plasmodium infected LLC cells is similar to that of the uninfected tumor cells but the major difference is in their functioning and the increase in granzyme B producing NK-cells within the infected tumor tissue was also examined.

Hence, the outcomes confirmed the role of plasmodium in the activation and elicitation of innate immune response within the suppressed tumor cells and as a result of that the enhanced functioning of NK-cells showed higher cytotoxicity to combat the tumor growth.

\section{Plasmodium as an Adjuvant Vaccine}

Adjuvants are the potent additives that play a vital role in the stimulation of immune response caused by antigens present in vaccine and persistence of long-lasting after effects. Several molecules found within the plasmodium parasite can be used in the development of an effective vaccine by acting as successful component i.e. adjuvant. ${ }^{[30]}$ For the evaluation of the plasmodium components whether they perform successfully as an adjuvant in a vaccine or not, plasmodium infected LLC cells were injected with either DNA vaccines (pcDNAhMUC1 and pcDNA3.1) to check the effect of plasmodium + DNA vaccine and the plain DNA vaccine over the tumor cells.

It was proved by the outcomes that the combination of DNA vaccine and plasmodium for treatment caused the synergistic effect in the tumor growth inhibition and prolonged the survival period. Hence, it was reported that the plasmodium can be used as a novel adjuvant for facilitation in assistance of tumor-specific antitumor immunity.

Immunotherapy have attracted the attention of all the scientists by playing a vital role in the cancer treatment that had been a major health issue for decades and neither any other therapeutic strategy nor the surgery was oftenly found to be that much successful and effective. The immunotherapy recovers suppressed immune response of the tumor host with the help of certain specific and non-specific immune stimulants. Conventional bio-immunoadjuvants like diphtheria, BCG and non-specific interventions including for stimulation of immune system i.e. TNF- $\alpha$, IFN- $\alpha$ and IL-2 that induce the inflammatory cells accumulation and Th1 type cytokines production which shows a significant positive effect over the tumor cells and simultaneously helps in the treatment. ${ }^{[7]}$ Whereas, the issue is with their immunity stimulation ability i.e. induce weak immune response, short-lasting and formation of defective memory cells. Hence, no desirable response was acquired by conventional clinical therapeutics unlike, certain recently developed pathogen-based immunotherapy. ${ }^{[40]}$

Table 4: Comparison between Conventional and Plasmodium-infected Adjuvants

\begin{tabular}{l|ll}
\hline Conventional adjuvants injected LLC Cells & & \multicolumn{1}{c}{ Plasmodium-infected LLC cells } \\
\hline - & Weak Immune Response & Strong and Tumor-Specific Immune Response \\
- Short-Lived & - & Long-Lasting \\
- Production of Defective Memory Cells & - & Production of Perfect Memory Cells \\
- Production of Non-tumor Specific Abs & - & Polyclonal Activation Induction \\
- Suppressed Immune System & - & Proliferation of Lymphocytes \\
\hline
\end{tabular}

\section{Cancer Treatment by GAG-Binding Malaria Protein}

With the invasion of Plasmodium falciparum in host body, it was examined that the parasite targets the erythrocytes (RBCs) at the first and starts to replicate within them. As they are now present within body, hence, they turns susceptible to the other RBCs as well and gets anchored to the particular cell receptors present in the vasculature of the host. ${ }^{[41]}$ The protein that helped in the anchorage was found to be VAR2CSA, it carries out the binding of RBCs to placental syncytiotrophoblasts. ${ }^{[42]}$ Placental malaria pathogenesis is the major event in which VAR2CSA interacts with the different type of chondroitin sulfate (CS) and glycosaminoglycan (GAG) polymer, collectively known as CSA. ${ }^{[3]}$ The CS-binding site of VAR2CSA have a $2 \mathrm{x}$ domian of DBL (Duffy binding ligand) with some interdomain (ID) flanking regions. The very domain interacts CS by significant specificity as well as affinity. ${ }^{[4]}$

By thorough examination of plasmodium infected RBCs, it was observed that their interaction with CS oligosaccharides took place by $4-O$-sulfated character. ${ }^{[4]}$ The parasites having VAR2CSA protein only do anchor to CS present in placenta despite of that they do not interacts elsewhere within body ${ }^{[42]}$ due to the distinctive CS subtype expression in placenta that was involved in cellular invasion and promotes rapid proliferation by being part of implantation phenomenon in placenta. ${ }^{[46]}$ Because of these two characteristics 
(Invasion and Proliferation) that are found similar in both plancenta and cancer, it was concluded that the similar CS may also play vital role in the treatment of cancer. Thats why, a recombinant protein of VAR2CSA (rVAR2), was produced for the targeting of cancerous cells of human. ${ }^{[41]}$ With the malarial infection, some genes (FabB/F, UIS3 and UIS4) starts to express within the RBCs at their pre-erythrocytic stage as they play an important role in the growth of infection. ${ }^{[4]}$ These genes were deleted from the host and the decline in infection growth was examined along with the infection avoidance. ${ }^{[48]}$

The malaria from this pre-erythrocytic stage was defective and is known as 'Genetically Attenuated Sporozoites' (GAS). Later on, it was reported that GAS was involved in the stimulation of immune response (IR) as former mentioned. ${ }^{[49]}$ It was concluded that these fluctuatuion could be proved beneficial in treating lung cancer. ${ }^{[50]}$ Human cancerous cells exhibit the similar expressed Placantal-Like CS. The ability of placental cells is associated with their chrondroitin sulfate proteoglycan (CSPG) component ${ }^{[51]}$ that maintains invasion of villous trophoblasts during implantation stage within uterine tissue and regulates the maximum proliferation rate. ${ }^{[46]}$ These are the phenotypic expressions that are correlated among the placental and tumor cells. It was reported that while having a clinical trial 95\% compatibility outcomes were obtained from recombinant rVAR2binding to the epithelial, mesenchymal and hematopoietic cancerous cell lines. This interation among rVAR2 and cells was highly based upon the protein concentration and if competed by CSA, it might get blocked. Moreover, $13 \mathrm{nM}$ is the KD-value of occurrence of higher affinity among C32 melanoma cells and rVAR2binding. On the other hand, VAR2CSA-secreting malarial infected RBCs were achored over surface of C32 cell line. This is the major key step that could be restricted by purified CSA.

ELISA and flow cytometry assays of the rVAR2-CSA were performed in order to evaluate the specificity with C32 cells. ${ }^{[41]}$ Only the human cancerous and placental trophoblast cells can produce such distinguished CS that can specifically be detected by VAR2CSA. The CSA-biosynthesis takes place with the involvement of several enzymes. ${ }^{[52]}$

\section{Malarial Genetically Attenuated Sporozoites as Potent Cancer Vaccine}

The application of genetically attenuated sporozoites (GAS) of malaria for the development of a potential vaccine in order to elicit immunization have been considered as an attractive alternative approach. Effect of GAS on the tumor growth was determined and the tumor-specific immunization was induced within tumor cells. After 14-days, the significant suppression in tumor growth was observed by measuring and comparing of the weights of tumor samples before and after particular time interval and the improvement in their survival period was also observed. This anti-cancer effect of GAS was studied thoroughly and it was acknowledged that the components of $1^{\text {st }}$ line of defense of innate immunity against metastatic invasion that activates the signaling pathway for the production of cytokines like IL-12/6, TNF- $\alpha$ and IFN- $\gamma$ were enhanced for provoking immediate immune response. ${ }^{[53]}$ By flow cytometry, peripheral blood mononuclear cells (PBMCs) were analyzed and the number of production levels of both T-lymphocytes (CD4+ and CD8+) were increased due to the application of genetically attenuated sporozoites (GAS).

\section{Plasmodium as Delivery Vector in Vaccine Development}

Most of the conventional vaccine vectors have been used intensively whereas, most of them successfully induces the cellular mediated immune response but their major drawback was that the induced immunity did not last for long, it was short-lived. Therefore, some other protozoans have also been studied after working on plasmodium such as, Toxoplasmosis gondii ${ }^{[54]}$ and Trypanosoma cruzi. ${ }^{[55]}$ For the development of a successful and effective antigen based tumor vaccine, a suitable delivery vector is needed. ${ }^{[56]}$

Jiang et al. Have worked previously on the plasmodium parasite as a delivery vector in order to develop an effective conceptual vaccine against human immunodeficiency virus (HIV) and it expressed the antitumor effect within mouse model while testing. ${ }^{[57]}$ Likewise, the plasmodium has been used as a delivery vector against a hepatocellular carcinoma (HCC) cancer type in a vaccine and showed significant results. The tumor growth that was inhibited due to plasmodium was the result of induced immune mechanisms.

A comparison among the Th1 and Th2-type cytokines was performed with and without the introduction of plasmodium into tumor cells and it was examined that the suppressed Th1-type cytokines (TNF- $\alpha$, IFN- $\gamma$ and IL2) started to increased in number and played a vital role in provoking immune response ${ }^{[58]}$ whereas, the Th2-type cytokines (IL-13, IL-5 and IL-4) remained unaffected and no change in the production rate was recorded. ${ }^{[59]}$ The cell-mediated immunity due to activity of cytotoxic T-lymphocytes (CD8+) was provoked in both of the stages of plasmodium life cycle i.e. in blood (Pre-erythrocytic stage) as well as in liver (Exo-erythrocytic stage) within the infected host exhibiting tumor. ${ }^{[60]}$ Plasmodium can be used as a delivery vector for the successful expression of ectopic tumor antigens at the pre-erythrocytic stage of infection. Within the duration of 2-3 weeks from the infection, the parasitemia within blood reaches upto 50 percent and starts to cure itself within another 2 weeks. Hence, this prolonged period for the continuous and gradual release of parasites is standardized for inducing the immunization. It is reported that the expression of GPC3 protein within parasities is similar to the tumor protein 
that regulates growth and proliferation of tumor cells and is responsible for the initiation of GPC-3 (TAA) specific immunity by the Cytotoxic T-lymphocytes (CTLs). It has been observed that major histocompatibility complex class I (MHC-I) is not present in the mature RBCs as well as immature infected RBCs (erythroblasts) thats why the association with phagocytes is required which might be the cause of inducing the immune responses by CD8+ T-cells that are MHC-I restricted. ${ }^{[60]}$ Hence, the induction of CD8+ CTL-based immune response is independent of the infected erythroblasts. It has been further studied that the malaria parasite at preerythrocytic stage induces CD8+ T-lymphocytes that are produced in response of antigens expressed by the parasite and are regulated by CD8 $\alpha+$ DCs. $^{[61]}$ The infection caused by Plasmodium falciparum and Plasmodium vivax was examined to be more likely in reticulocytes. ${ }^{[62]}$ The changes in physiological and immunological processes due to plasmodium experienced in mice are supposed to be the similar within humans that could be of immense significance and puropose while treating the any kind of tumor.

According to the previous hypothesis, it was assumed that the activation of CD4+ Th1-type-based immune reponse takes place at the first which later on triggers strong and lost-lasting CTL that is GPC-specific. A vaccine was designed consisting of plasmodium and GPC3 antigen that enhances the immunological processes of the host in order to counteract tumor cells growth. Indeed it may suppress the growth of tumor cells but can not eliminate these cells completely. That incomplete elimination was discovered to be because of small fraction of GPC3-antigen expressing tumor cells. It was concluded that a novel immunotherapy for the treatment of cancer was developed that involve use of plasmodium parasite as a delivery vector for the expression of tumorassociated antigens (TAA) that would ultimately elicit the tumor-suppressed immune response. The immunomodulation of the immune system would be outcome of action of this vaccine. By experimental trial, the prolonged survival period as well as the enhanced immune system was reported within the mice models. Hence, the attenuated parasites as well as their attenuated sporozoites can be used as live vectors in vaccine development. ${ }^{[63]}$

\section{GAS induced Immunization}

Innate and adaptive immunity are the two major lines of defenses in the immune system of an organism. Innate immune system is usually the general and resistant for all the antigens whereas, adaptive is variable and antigenspecific that regulates the immunity. For the activation of adaptive immune system, signals from the innate immune system are needed which shows that the innate immune system regulates the adaptive immune system. ${ }^{[64]}$ The innate immunity in the tumor affected patients was immunomodulated by the introduction of attenuated sporozoites of plasmodium that stimulates the production of secretions of humoral immunity i.e. IL12 , IL-6, TNF- $\alpha$ and IFN- $\gamma$ within the three days of GAS insertion. These cytokines are the part of humoral immunity of innate immune system that are required for the induction of specific T-lymphocytes production that would result in activation of adaptive immunity and shows antitumor immune response. ${ }^{[65]}$ Such immunization results in the strong adaptive immune response that would be cellular with the help of T-lymphocytes activity and proliferation against tumor cells. ${ }^{[49]}$ It has been reported that genetically attenuated sporozoites (GAS) induced systemin immunity lasts for a long time interval and also produces memory cells.

\section{Immunosuppression by Tumor and Tumor-Specific Immune Response Provoked by Malaria}

Immunosurveillance is the mechanism of the scaning and monitoring of entire body for the detection of neoplastic or malignant cells that express particular tumor antigens. After recognition of such cells, the immune system of body takes it as a foreign particle or antigen and tries to destroy them. ${ }^{[66]}$ In contrast to this, tumor cells exhibit the capability of suppressing the host immune system (immunosuppression) by activating immunological brakes or inhibitory feedback loopsor (Figure 1). Another function of these immunological brakes is avoidance of auto-immune disorders, creating barriers for activation of T-cells and tumor rejection. ${ }^{[67]}$ Hence, the recovery of host immune response from the tumor induced immunosuppression and tolertogenic status for the cancer therapeutics is convenient. 


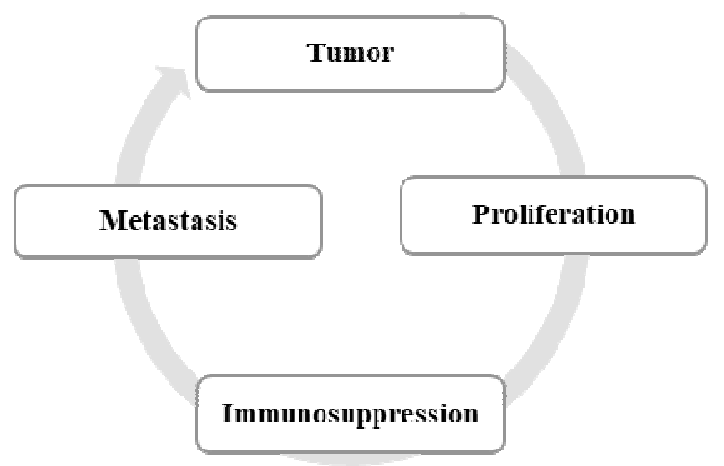

Fig. 1: Immunosuppression Induced by Tumor

There are various similar characteristics among parasites and cancer types as both of them weakens/suppresses the host immun system, harms at cellular, tissue or organ levels, develops inside the host and may metastasize at other locations within host. ${ }^{[68]}$ It has been reported that avoidance of immunosurveillance and interation with innate immune system is acquired by the both (Parasites and Cancer cells) for the sake of development of their successful colonization. Therefore, due to this resemblance, the application of anti-parasitic drug have been assumed as advantageous in treatment of cancer as it may also act as anti-cancer drug. ${ }^{69]}$ Likewise, it has been studied that parasites might show antitumor activity by inducing immunomodulation (Figure 2). ${ }^{[54]}$

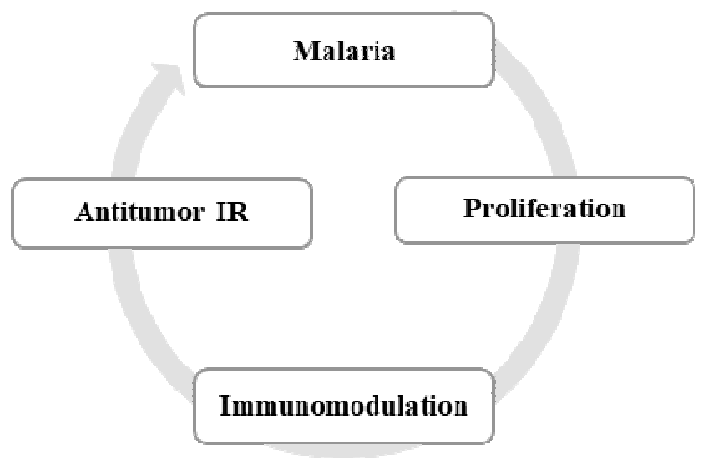

Fig. 2: Immunomodulation Induced by Plasmodium

\section{Limitations of Plasmodium Immunotherapeutic Vaccine}

It has been acknowledged that most of the trials that have been performed were on rodents that shows ofcourse great similarity with human but certain complications were observed such as, there was no fever suffering (Hyperthermia) due to malaria in rodents unlike human ${ }^{[70]}$ and the antitumor effects are more long-lasting in rodents, upto a month, which illustrates that infectious period is directly proportional to the effect on tumor growth inhibition. While the benign malaria in human was observed to cause hyperthermia at acute phase and the malaria parasite could survie for years in chronic stages if the treatment is not provided. ${ }^{[71]}$

\section{Current Status and Future Considerations}

The Plasmodium parasite has been used as the vaccine component due to its antitumor activity by resembling with cancer in characteristics and antigens in certain clinical trials. Certain other protozoans have also been studied after this therapeutic breakout of plasmodium and some of them have also been found as potential vectors and adjuvants for the vaccine development. But the research does not stops, scientists keep on digging the most advanced strategies for the ease in the treatment of health disasters like cancer in order to control its high mortality rate and protect the human health. Though the use of microbes in vaccine development is a traditional strategy by the process of attenuation but the plasmodium was used for the first time and its incredible antitumor effects have been observed. In near future, it is being anticipated that this immunotherapeutic agent would dominate other cancer treating strategies due to its higher efficiency as well as the biosecurity due to targeted treatment. The research on this approach regarding biotechnological applications have also been under processing as it's the era of bionanotechnology. 


\section{References}

1. CDC, Malaria. U.S. Centers for Disease Control and Prevention, 2015.

2. Jemal, A., et al., Cancer statistics, 2010. CA: a cancer journal for clinicians, 2010. 60(5): p. 277-300.

3. Ferlay, J., et al., Cancer incidence and mortality worldwide: sources, methods and major patterns in GLOBOCAN 2012. International journal of cancer, 2015. 136(5): p. E359-E386.

4. Kelly, R.J., J.L. Gulley, and G. Giaccone, Targeting the immune system in non-small-cell lung cancer: bridging the gap between promising concept and therapeutic reality. Clinical lung cancer, 2010. 11(4): p. 228-237.

5. Decoster, L., I. Wauters, and J. Vansteenkiste, Vaccination therapy for non-small-cell lung cancer: review of agents in phase III development. Annals of oncology, 2012. 23(6): p. 1387-1393.

6. Baird, J.R., et al., Immune-mediated regression of established B16F10 melanoma by intratumoral injection of attenuated Toxoplasma gondii protects against rechallenge. The Journal of Immunology, 2013. 190(1): p. 469-478.

7. Van den Heuvel, M.M., S.A. Burgers, and N. van Zandwijk, Immunotherapy in non-small-cell lung carcinoma: from inflammation to vaccination. Clinical lung cancer, 2009. 10(2): p. 99-105.

8. Paulides, M., et al., The clinical feasibility of deep hyperthermia treatment in the head and neck: new challenges for positioning and temperature measurement. Physics in medicine and biology, 2010. 55(9): p. 2465.

9. Ing, R., et al., Interaction of mouse dendritic cells and malaria-infected erythrocytes: uptake, maturation, and antigen presentation. The Journal of Immunology, 2006. 176(1): p. 441-450.

10. Roetynck, S., et al., Natural killer cells and malaria. Immunological reviews, 2006. 214(1): p. 251-263.

11. Kalinski, P., et al., Natural killer-dendritic cell cross-talk in cancer immunotherapy. Expert opinion on biological therapy, 2005. 5(10): p. 1303-1315.

12. Zhang, H.-G., et al., Hyperthermia on immune regulation: a temperature's story. Cancer letters, 2008. 271(2): p. 191-204.

13. Chen, L., et al., Antitumor Effect of Malaria Parasite Infection in a Murine Lewis Lung Cancer Model through Induction of. 2011

14. Mittal, S. and H.B. El-Serag, Epidemiology of HCC: consider the population. Journal of clinical gastroenterology, 2013. 47: p. S2.

15. Siegel, R.L., K.D. Miller, and A. Jemal, Cancer statistics, 2015. CA: a cancer journal for clinicians, 2015. 65(1): p. 5-29.

16. Sawada, Y., et al., Phase I trial of a glypican-3-derived peptide vaccine for advanced hepatocellular carcinoma: immunologic evidence and potential for improving overall survival. Clinical Cancer Research, 2012. 18(13): p. 3686-3696.

17. Lee, W.-C., et al., Vaccination of advanced hepatocellular carcinoma patients with tumor lysate-pulsed dendritic cells: a clinical trial. Journal of Immunotherapy, 2005. 28(5): p. 496-504.

18. Din, M.O., et al., Synchronized cycles of bacterial lysis for in vivo delivery. Nature, 2016. 536(7614): p. 8185 .

19. Jaramillo, M., et al., Hemozoin-inducible proinflammatory events in vivo: potential role in malaria infection. The Journal of Immunology, 2004. 172(5): p. 3101-3110.

20. Krishnegowda, G., et al., Induction of proinflammatory responses in macrophages by the glycosylphosphatidylinositols of Plasmodium falciparum cell signaling receptors, glycosylphosphatidylinositol (GPI) structural requirement, and regulation of GPI activity. Journal of Biological Chemistry, 2005. 280(9): p. 8606-8616.

21. Stevenson, M.M. and E.M. Riley, Innate immunity to malaria. Nature Reviews Immunology, 2004. 4(3): p. 169-180.

22. Iwama, $\mathrm{T}$., et al., Identification of an $\mathrm{H} 2-\mathrm{Kb}$ or $\mathrm{H} 2-\mathrm{Db}$ restricted and glypican-3-derived cytotoxic $\mathrm{T}$ lymphocyte epitope peptide. International journal of oncology, 2013. 42(3): p. 831-838.

23. Sawada, Y., et al., Phase II study of the GPC3-derived peptide vaccine as an adjuvant therapy for hepatocellular carcinoma patients. Oncoimmunology, 2016. 5(5): p. e1129483.

24. Gao, H., et al., Development of $\mathrm{T}$ cells redirected to glypican-3 for the treatment of hepatocellular carcinoma. Clinical Cancer Research, 2014. 20(24): p. 6418-6428.

25. Nakatsura, T., et al., Glypican-3, overexpressed specifically in human hepatocellular carcinoma, is a novel tumor marker. Biochemical and biophysical research communications, 2003. 306(1): p. 16-25.

26. Sehrawat, N. and A. Sharma, Malaria: A Concern to Public Health. SHODH PRERAK, 2012: p. 194.

27. Matteelli, A., F. Castelli, and S. Caligaris, Life cycle of malaria parasites. Handbook of Malaria Infection in the Tropics, 1st edn. Associazione Italiana "Amici di R. Follereau": Bologna, 1997: p. 17-23.

28. Mueller, I., et al., Key gaps in the knowledge of Plasmodium vivax, a neglected human malaria parasite. The Lancet infectious diseases, 2009. 9(9): p. 555-566. 
29. Donati, D., et al., Identification of a polyclonal B-cell activator in Plasmodium falciparum. Infection and immunity, 2004. 72(9): p. 5412-5418.

30. Coban, C., et al., TLR9 and endogenous adjuvants of the whole blood-stage malaria vaccine. Expert review of vaccines, 2010. 9(7): p. 775-784.

31. Wood, L.M., et al., Cancer immunotherapy using Listeria monocytogenes and listerial virulence factors. Immunologic research, 2008. 42(1-3): p. 233-245.

32. Tangney, M. and C.G. Gahan, Listeria monocytogenes as a vector for anti-cancer therapies. Current gene therapy, 2010. 10(1): p. 46-55.

33. Bulk, E., et al., Adjuvant therapy with small hairpin RNA interference prevents non-small cell lung cancer metastasis development in mice. Cancer research, 2008. 68(6): p. 1896-1904.

34. Steeg, P.S., Tumor metastasis: mechanistic insights and clinical challenges. Nature medicine, 2006. 12(8): p. 895 .

35. Kamoun, W.S., et al., Edema control by cediranib, a vascular endothelial growth factor receptor-targeted kinase inhibitor, prolongs survival despite persistent brain tumor growth in mice. Journal of clinical oncology, 2009. 27(15): p. 2542-2552.

36. Tanaka, H., et al., Successful adoptive immunotherapy of murine poorly immunogenic tumor with specific effector cells generated from gene-modified tumor-primed lymph node cells. The Journal of Immunology, 1999. 162(6): p. 3574-3582.

37. Boissonnas, A., et al., Foxp3+ T cells induce perforin-dependent dendritic cell death in tumor-draining lymph nodes. Immunity, 2010. 32(2): p. 266-278.

38. Kurts, C., B.W. Robinson, and P.A. Knolle, Cross-priming in health and disease. Nature Reviews Immunology, 2010. 10(6): p. 403-414.

39. Ullrich, E., et al., Dendritic cells and innate defense against tumor cells. Cytokine \& growth factor reviews, 2008. 19(1): p. 79-92.

40. Alexandroff, A.B., et al., BCG immunotherapy of bladder cancer: 20 years on. The Lancet, 1999. 353(9165): p. 1689-1694.

41. Salanti, A., et al., Targeting human cancer by a glycosaminoglycan binding malaria protein. Cancer cell, 2015. 28(4): p. 500-514.

42. Salanti, A., et al., Evidence for the involvement of VAR2CSA in pregnancy-associated malaria. Journal of Experimental Medicine, 2004. 200(9): p. 1197-1203.

43. Dahlbäck, M., et al., The chondroitin sulfate A-binding site of the VAR2CSA protein involves multiple Nterminal domains. Journal of Biological Chemistry, 2011. 286(18): p. 15908-15917.

44. Clausen, T.M., et al., Structural and functional insight into how the Plasmodium falciparum VAR2CSA protein mediates binding to chondroitin sulfate A in placental malaria. Journal of Biological Chemistry, 2012. 287(28): p. 23332-23345.

45. Beeson, J.G., et al., Structural basis for binding of Plasmodium falciparum erythrocyte membrane protein 1 to chondroitin sulfate and placental tissue and the influence of protein polymorphisms on binding specificity. Journal of Biological Chemistry, 2007. 282(31): p. 22426-22436.

46. Baston-Büst, D.M., et al., Syndecan-1 knock-down in decidualized human endometrial stromal cells leads to significant changes in cytokine and angiogenic factor expression patterns. Reproductive Biology and Endocrinology, 2010. 8(1): p. 133.

47. Vaughan, A., R. Wang, and S.H. Kappe, Genetically engineered, attenuated whole-cell vaccine approaches for malaria. Human vaccines, 2010. 6(1): p. 107-113.

48. VanBuskirk, K.M., et al., Preerythrocytic, live-attenuated Plasmodium falciparum vaccine candidates by design. Proceedings of the National Academy of Sciences, 2009. 106(31): p. 13004-13009.

49. Seder, R.A., et al., Protection against malaria by intravenous immunization with a nonreplicating sporozoite vaccine. Science, 2013. 341(6152): p. 1359-1365.

50. Deng, X.-F., et al., Antitumor effect of intravenous immunization with malaria genetically attenuated sporozoites through induction of innate and adaptive immunity. International Journal of Clinical and Experimental Pathology, 2016. 9(2): p. 978-986.

51. Van Sinderen, M., et al., The chrondroitin sulfate proteoglycan (CSPG4) regulates human trophoblast function. Placenta, 2013. 34(10): p. 907-912.

52. Sugahara, K., et al., Recent advances in the structural biology of chondroitin sulfate and dermatan sulfate. Current opinion in structural biology, 2003. 13(5): p. 612-620.

53. Benito-Martin, A., et al., The new deal: a potential role for secreted vesicles in innate immunity and tumor progression. Novel clinical applications of extracellular vesicles, 2015: p. 19.

54. Kim, J.-O., et al., Inhibition of Lewis lung carcinoma growth by Toxoplasma gondii through induction of Th1 immune responses and inhibition of angiogenesis. Journal of Korean medical science, 2007. 22(Suppl): p. S38-S46. 
55. Junqueira, C., et al., Trypanosoma cruzi as an effective cancer antigen delivery vector. Proceedings of the National Academy of Sciences, 2011. 108(49): p. 19695-19700.

56. Liu, M.A., Immunologic basis of vaccine vectors. Immunity, 2010. 33(4): p. 504-515.

57. Jiang, B., et al., Transgenic Plasmodium that expresses HIV-1 Gag elicits immunity and protects mice against vaccinia virus-gag and malarial parasites. Vaccine, 2010. 28(50): p. 7915-7922.

58. Liu, Q., et al., Plasmodium parasite as an effective hepatocellular carcinoma antigen glypican-3 delivery vector. Oncotarget, 2017. 8(15): p. 24785.

59. Gazzinelli, R.T. and E.Y. Denkers, Protozoan encounters with Toll-like receptor signalling pathways: implications for host parasitism. Nature Reviews Immunology, 2006. 6(12): p. 895-906.

60. Imai, T., et al., Cytotoxic activities of CD8+ T cells collaborate with macrophages to protect against bloodstage murine malaria. Elife, 2015. 4: p. e04232.

61. Lundie, R.J., et al., Blood-stage Plasmodium infection induces CD8+ T lymphocytes to parasite-expressed antigens, largely regulated by $\mathrm{CD} 8 \alpha+$ dendritic cells. Proceedings of the National Academy of Sciences, 2008. 105(38): p. 14509-14514.

62. Lim, C., et al., Reticulocyte Preference and Stage Development of Plasmodium vivax Isolates. The Journal of infectious diseases, 2016. 214(7): p. 1081-1084.

63. Deng, X., et al., Antitumor effect of intravenous immunization with malaria genetically attenuated sporozoites through induction of innate and adaptive immunity. Int J Clin Exp Pathol, 2016. 9(2): p. 978-86.

64. Iwasaki, A. and R. Medzhitov, Control of adaptive immunity by the innate immune system. Nature immunology, 2015. 16(4): p. 343-353.

65. Tanaka, M., et al., Induction of antitumor immunity by combined immunogene therapy using IL-2 and IL-12 in low antigenic Lewis lung carcinoma. Cancer gene therapy, 2000. 7(11): p. 1481.

66. Domingues, D., et al., Immunotherapy and lung cancer: current developments and novel targeted therapies. 2014.

67. Schreiber, R.D., L.J. Old, and M.J. Smyth, Cancer immunoediting: integrating immunity's roles in cancer suppression and promotion. Science, 2011. 331(6024): p. 1565-1570.

68. Psaila, B. and D. Lyden, The metastatic niche: adapting the foreign soil. Nature Reviews Cancer, 2009. 9(4): p. 285-293.

69. Van Huijsduijnen, R.H., et al., Anticancer properties of distinct antimalarial drug classes. PLoS One, 2013. 8(12): p. e82962.

70. Iwalewa, E. and E. Agbani, Effects of autacoid inhibitors and of an antagonist on malaria infection in mice. Brazilian journal of medical and biological research, 2004. 37(8): p. 1199-1204.

71. Song, H.H., et al., Clinical features of Plasmodium vivax malaria. The Korean journal of internal medicine, 2003. 18(4): p. 220. 